\title{
Perimenopausal Bleeding a Risk Factor for Endometrial Hyperplasia and Endometrial Cancer
}

\section{Wirun Thongchumnum ${ }^{1}$, Sakda Arj-Ong Vallibhakara ${ }^{2,3}$, Areepan Sophonsritsuk ${ }^{4}$ and Orawin Vallibhakara ${ }^{4 *}$}

${ }^{1}$ Department of Obstetrics and Gynaecology, Faculty of Medicine, Ramathibodi Hospital, Mahidol University, Bangkok, Thailand

${ }^{2}$ ASEAN Institute for Health Development (AiHD), Mahidol University, Nakhon

Pathom, Thailand

${ }^{3}$ Department of Clinical Epidemiology and Biostatistics, Faculty of Medicine,

Ramathibodi Hospital, Mahidol University, Bangkok, Thailand

${ }^{4}$ Reproductive Endocrinology and Infertility Unit, Department of Obstetrics and

Gynaecology, Faculty of Medicine, Ramathibodi Hospital, Mahidol University,

Bangkok, Thailand

*Corresponding Author: Orawin Vallibhakara, Reproductive Endocrinology and Infertility Unit, Department of Obstetrics and Gynaecology, Faculty of Medicine, Ramathibodi Hospital, Mahidol University, Bangkok, Thailand.

DOI: $10.31080 /$ ASWH.2020.02.0133
Received: June 15, 2020

Published: July 31, 2020

(C) All rights are reserved by Orawin

Vallibhakara., et al.

\section{Abstract}

Objectives: To determine the etiologies, patterns of bleeding and the associated factors of endometrial hyperplasia and cancer in perimenopausal women.

Methods: A cross-sectional research approach was used in this study. Women $(\mathrm{n}=346)$ with perimenopausal abnormal uterine bleeding (AUB) visited the Department of Obstetrics and Gynecology, Ramathibodi Hospital, Mahidol University, Thailand, a tertiary medical school were enrolled in this study. Ethical clearance was obtained from the University's human ethics committee. Data collection included history taking, pelvic examination, transvaginal sonography, and endometrial pathology. Data analysis was through descriptive and inferential statistics the factors which had statistically significant (p-value $<0.10)$ were included in multiple logistic regression analysis to evaluate the significant factors related to endometrial hyperplasia and cancer.

Results: Among all the participants $(\mathrm{n}=346) 164$ were indicated for gynecologic surgery $(47.4 \%)$. Causes of bleeding included fibroids (31.21\%) and adenomyosis (24.28\%), whereas the prevalence of endometrial hyperplasia and cancer was $12.5 \%$. The common perimenopausal bleeding patterns were intermenstrual bleeding (IMB) (47.69\%) and heavy menstrual bleeding (HMB) (47.11\%). After the univariate and multiple logistic regression analyses, the significant risk factors of endometrial hyperplasia and cancer were endometrial thickness > 10 mm (OR 7.61, 95\%CI 3.52 - 16.45) and IMB (OR 4.66, 95\%CI 2.05 - 10.57), respectively. Conclusion: The uterine fibroids and adenomyosis took a majority of perimenopausal AUB causes, which mostly presenting with IMB or HMB patterns. However, endometrial hyperplasia and cancer should be concerned, especially in present AUB, with evidence of thick endometrium or IMB.

Keywords: Perimenopausal Bleeding; Abnormal Uterine Bleeding; Endometrial Hyperplasia; Endometrial Cancer; Perimenopausal Woman

\section{Abbreviations}

AUB: Abnormal Uterine Bleeding; DM: Diabetic Mellitus; EIN: Endometrial Intraepithelial Neoplasia; E2: Estradiol; FIGO: The International Federation of Gynaecology and Obstetrics; FSH: Follicular Stimulating Hormone; HMB: Heavy Menstrual Bleeding; HPMB: Heavy and Prolonged Menstrual Bleeding; IMB: Intermenstrual Bleeding; MUSA: Morphological Uterus Sonographic Assessment; OR: Odd Ratios

\section{Introduction}

Abnormal uterine bleeding (AUB) is a common problem, accounting for $30 \%$ of the gynecological outpatient department [1] since the bleeding effects on the quality of life and is one of the warning signs of the female reproductive system diseases. According to the International Federation of Gynaecology and Obstetrics (FIGO) 2011 announcement, AUB pattern could be classified in terms of heavy menstrual bleeding (HMB), intermenstrual bleed- 
ing (IMB) and heavy and prolonged menstrual bleeding (HPMB) [2]. Alongside, the polyp; adenomyosis; leiomyoma; malignancy and hyperplasia; coagulopathy; ovulatory dysfunction; endometrial; iatrogenic; and not yet classified (PALM-COEIN) system categorized the etiologies of bleeding [3].

The AUB is also one of the commonest problems of perimenopausal women defined at age around 40 or a period of menstrual abnormalities. From a physiologic change of menopausal transition, the depletion of ovarian follicles results in cycle irregularities, followed by an AUB [4]. Despite hormonal imbalance seem to be the direct cause of AUB among perimenopausal women [5], the bleeding either from anatomical causes or local endometrial pathology must be excluded, especially endometrial hyperplasia and cancer [6]. Endometrial cancer is the most common gynecologic malignancy in developed countries with an incidence of 23.2/100,000 women in the United States. While in most developing countries of Africa and Asia, including Thailand, endometrial cancer is the second most common gynecological cancer after cervical cancer, with an incidence of fewer than 5/100,000 women [7,8]. Although most cases are postmenopausal women, around 17 percent of endometrial cancer is detected during the perimenopausal period [9]. The main risk factor of endometrial cancer is an unopposed exposure to estrogens, either from endogenous or exogenous sources that associated with obesity, diabetes, early age at menarche, nulliparity, late-onset menopause, older age and tamoxifen use [10].

\section{Aim of the Study}

This study aims to define the etiologies of AUB in the perimenopausal women, the patterns of bleeding, and the factors associated with endometrial hyperplasia and cancer.

\section{Materials and Methods}

\section{Study design}

A cross-sectional study of perimenopausal women, who presented with AUB and visited the Department of Obstetrics and Gynecology, Ramathibodi Hospital, Mahidol University, Thailand, a tertiary medical school, was performed between $1^{\text {st }}$ March 2018 and $31^{\text {st }}$ January 2019 . The study was approved by the Ethical Clearance Committee on Human Rights Related to Researches Human Subjects, Faculty of Medicine, Ramathibodi Hospital, Mahidol University (MURA2017/845). The primary outcome was to identify etiologic characteristics among perimenopausal bleeding women. The secondary outcomes were to discover the clinical presentation of the bleeding pattern and risk factors associated with endometrial hyperplasia and endometrial cancer in perimenopausal women presenting with AUB.

\section{Participants}

Perimenopausal women presented with AUB were enrolled in the study. The inclusion criteria were women age between 40 and
55 who present with any AUB, including heavy menstrual bleeding (HMB), intermenstrual bleeding (IMB) and heavy and prolonged menstrual bleeding (HPMB), whose endometrial pathology were obtained. All participants provided an informed consent. The women were excluded in case of menopause, which defined by a history of amenorrhea for more than 12 months or exceeded serum follicular stimulating hormone (FSH) level more than $40 \mathrm{IU} / \mathrm{ml}$. The other exclusion criteria were history of endometrial cancer, ongoing pregnancy, coagulopathy, currently taking anti-coagulant medications or hormonal contraception or hormonal therapy, intrauterine device use, bleeding from lower genital tract lesions such as cervical polyp or cervical cancer and inadequate endometrial tissue evaluation. Eventually, from 349 perimenopausal women, 346 were included in the study and three patients were excluded, as shown in the protocol flow diagram (Figure 1).

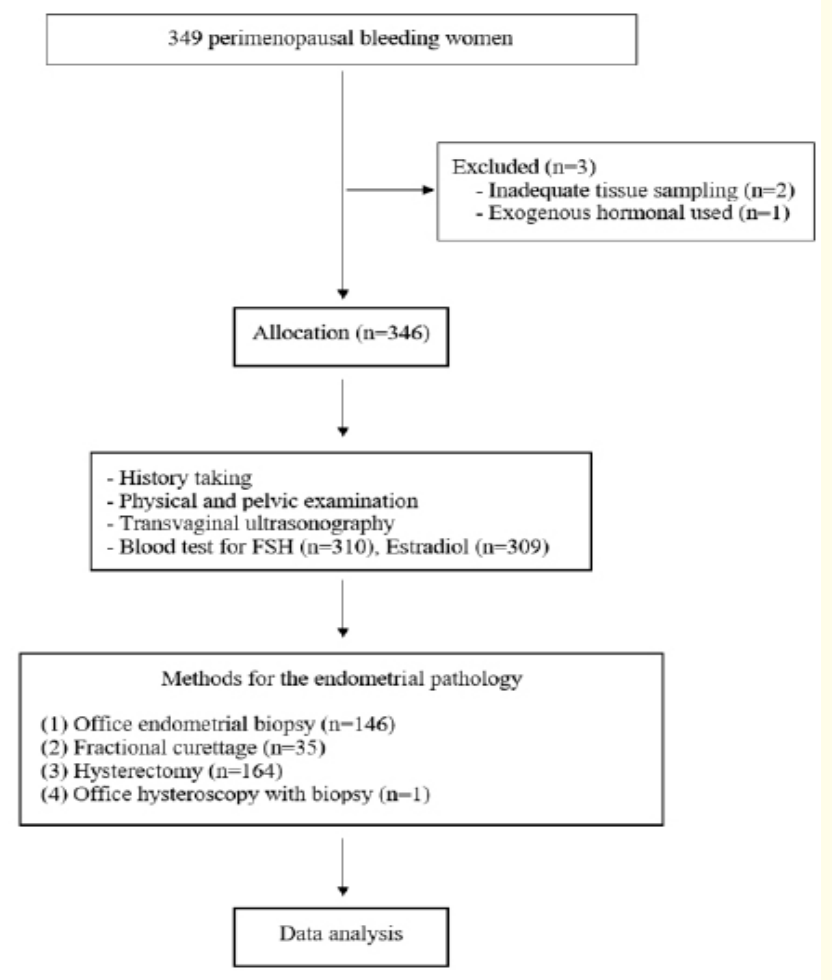

Figure 1: The protocol flow diagram of 349 perimenopausal women presenting with AUB.

\section{Data collection and measurements}

All participants were examined and their information was collected about the bleeding characteristics, parity, underlying disease, current medication, family history of any cancer, and personal history. Bleeding patterns were categorized based on FIGO recommendation (2) as 1) heavy menstrual bleeding (HMB): an excessive menstrual blood loss which interferes with the woman's physical, emotional, social and quality of life, 2) intermenstrual 
bleeding (IMB): an irregular episodes of bleeding occur between otherwise fairly normal menstrual periods and 3) heavy and prolonged menstrual bleeding (HPMB) which mean HMB with duration at least 8 days. Secondly, pelvic examination was performed to either identify the cause of bleeding or exclusion of the lower genital tract lesions. Afterward, pelvic ultrasonography was done by 4 - 9 MHz bandwidth transvaginal probe model EV4-9/10ED of Samsung Medison SonoAce R7 (Samsung Medison CO., LTD., Gangnam-gu, Seoul, Korea). The ultrasonography was systematically performed starting at mid-sagittal plane of the uterus to evaluate either myometrium or endometrium lesion. Endometrial thickness was measured by maximum width of the endometrial echo in the sagittal plane. Then, the axial plane of uterus and both adnexa were evaluated. Finally, we perform one of four methods for endometrial histopathological examination according to the factors and indications of each patient. Those methods were office endometrial biopsy, fractional curettage under general anesthesia, office hysteroscopy with biopsy, and indicated hysterectomy. Moreover, most of the participants were investigated for the serum follicular stimulating hormone (FSH) level and serum estradiol (E2) level to correlate with the menopausal status. After receiving all of the clinical information and investigations, the final diagnosis or the etiologies of the bleeding were discussed among consensus of certified gynecologist investigators.

\section{Statistical analysis}

Baseline characteristics were reported as descriptive data. All quantitative variables were tested for normal distribution. Mean (standard deviation, SD) and median (range) was used when data were normal and non-normal distribution, respectively. The statistical analysis was performed by STATA Version 15.0 (College Station TX, USA). The risk factors for endometrial hyperplasia and endometrial cancer were analyzed with univariate analysis. If they found a significant association of factors ( $p$-value $<0.10$ ), the multiple logistic regression was used to evaluate the independent factor effect. The statistical significance in our study was defined when P-value $<0.05$ together with $95 \%$ confidence intervals (CI).

\section{Results and Discussion}

Baseline characteristics of 346 perimenopausal women, the mean age was $46.38( \pm 3.66)$ years old. Most participants were multiparous (67.63\%) and no history of contraceptive use (58\%). Some participants had underlying diseases, including hypertension (12.43\%), dyslipidemia (8.96\%), and diabetes (4.91), see table 1 . The common bleeding patterns were intermenstrual bleeding (IMB) (47.69\%) and heavy menstrual bleeding (HMB) (47.11\%), while the heavy and prolonged menstrual bleeding was found only $5.2 \%$. After the investigator performed the pelvic examination and transvaginal sonography, the measurement of en- dometrial thickness was also evaluated in most participants except for 44 participants who have endometrial distortion effected on the accuracy of the measurement. The endometrial pathologies were obtained from 146 office-endometrial biopsies, 35 fractional curettages under general anesthesia, 164 indicated hysterectomy including both laparotomy and laparoscopic surgery, and one office hysteroscopy with biopsy. The etiologies of the perimenopausal bleeding are shown in table 2. Uterine fibroids and adenomyosis were responsible for more than half of perimenopausal bleeding, $30.92 \%$ and $24.28 \%$, respectively. Only submucous fibroids and huge intramural fibroids, which size more than $4 \mathrm{~cm}$ in diameters, from either sonographic or pathologic findings, were diagnosed uterine fibroids for the etiology of the bleeding. Adenomyosis was diagnosed based on clinical presentation, physical findings of the enlarged globular-shaped uterus, sonographic findings according to morphological uterus sonographic assessment (MUSA) criteria [11], or pathological report from the hysterectomy.

\begin{tabular}{|c|c|}
\hline Characteristics & $\mathrm{N}=346(\%)$ \\
\hline Age (years) ${ }^{\mathrm{a}}$ & $46.48 \pm 3.66$ \\
\hline Body mass index $\left(\mathrm{kg} / \mathrm{m}^{2}\right)$ & $24.32(17.58,49.45)$ \\
\hline Menarche (years) ${ }^{\mathrm{a}}$ & $13.68 \pm 1.59$ \\
\hline Waist circumference $(\mathrm{cm})^{\mathrm{a}}$ & $79.05 \pm 8.51$ \\
\hline \multicolumn{2}{|l|}{ Parity $(\mathrm{N})^{\mathrm{c}}$} \\
\hline Nulliparous & $112(32.37)$ \\
\hline Multiparous & $234(67.63)$ \\
\hline \multicolumn{2}{|l|}{$\begin{array}{l}\text { History of contraceptive meth- } \\
\text { ods }(\mathrm{N})^{\mathrm{c}}\end{array}$} \\
\hline Tubal ligation & $103(29.76)$ \\
\hline Condom & $20(5.78)$ \\
\hline Hormonal contraception & $15(4.34)$ \\
\hline Intrauterine device & $2(0.58)$ \\
\hline \multicolumn{2}{|l|}{ Underlying disease $(\mathrm{N})^{\mathrm{c}}$} \\
\hline Hypertension & $43(12.43)$ \\
\hline Dyslipidemia & $31(8.96)$ \\
\hline Diabetic Mellitus & $17(4.91)$ \\
\hline Breast cancer & $11(3.18)$ \\
\hline \multicolumn{2}{|l|}{ Serum hormone levels ${ }^{b}$} \\
\hline FSH (n = 310) (IU/dL) & $7.53(0.42,295.7)$ \\
\hline Estradiol $(\mathrm{n}=309)(\mathrm{pg} / \mathrm{mL})$ & $55.9(4,840.9)$ \\
\hline $\begin{array}{l}\text { Endometrial thickness }^{\mathrm{b}} \\
(\mathrm{n}=302)(\mathrm{mm})\end{array}$ & $7(1.2,63.6)$ \\
\hline
\end{tabular}

Table 1: Participants' baseline characteristics.

${ }^{\mathrm{a}}$ Data expressed as mean \pm standard deviation (SD). ${ }^{\mathrm{b}}$ Data expressed as median (range). ${ }^{c}$ Data expressed as a percentage. FSH: Follicular Stimulating Hormone. 


\begin{tabular}{|l|c|}
\hline \multicolumn{1}{|c|}{ Etiologies of bleeding } & N = 346 (\%) \\
\hline Fibroids & $108(31.21)$ \\
\hline Adenomyosis & $84(24.28)$ \\
\hline Endometrial polyp & $61(17.63)$ \\
\hline Hormonal imbalance & $43(12.43 \%)$ \\
\hline Endometritis & $5(1.44 \%)$ \\
\hline Endometrial atrophy & $1(0.29 \%)$ \\
\hline Pre-malignant and malignant endometrium & $44(12.72)$ \\
\hline Benign hyperplasia (BEH) & $22(6.36)$ \\
\hline Endometrial intraepithelial neoplasia (EIN) & $10(2.89)$ \\
\hline Endometrial cancer & $12(3.47 \%)$ \\
\hline
\end{tabular}

Table 2: Etiologies of perimenopausal bleeding. Data expressed as a percentage.
Endometrial hyperplasia and endometrial cancer were found in $12.72 \%$, in which the prevalence of endometrial cancer was 12 of 44 patients $(3.47 \%)$. From the univariate analysis, the significant risk factors of endometrial hyperplasia and cancer were diabetic mellitus (DM), IMB and endometrial thickness $\geq 10 \mathrm{~mm}$ from transvaginal sonography. The thickening of endometrium had the strongest correlation (Odd Ratios (OR) 7.5, 95\% CI: 3.52-17.4), followed by IMB (OR $=4.84,95 \% \mathrm{CI}: 2.1-11.1)$ and DM (OR $=4.17,95 \% \mathrm{CI}$ : 1.44-12.11) as shown in table 3 . We performed multiple logistic regression analysis among significant factors which significant $\mathrm{p}$ value was below 0.10 . The endometrial thickness $\geq 10 \mathrm{~mm}$ from sonographic findings (OR $=7.61,95 \% \mathrm{CI}: 3.52-16.45$ ) and IMB pattern (OR $=4.66,95 \% \mathrm{CI}: 2.05-10.57)$ were the only two significant factors associated with endometrial hyperplasia and cancer in perimenopausal women presenting with AUB, see table 4.

\begin{tabular}{|c|c|c|c|c|c|}
\hline Factors & Non-BEH N (\%) & $\mathrm{BEH}^{+} \mathrm{N}(\%)$ & OR & $95 \% \mathrm{CI}$ & P-value \\
\hline \multicolumn{6}{|l|}{ Age (years) } \\
\hline $40-45$ & 125 (87.41) & $18(12.59)$ & 1 & & 0.84 \\
\hline $46-50$ & $132(88)$ & $18(12)$ & 0.95 & $0.47-1.9$ & \\
\hline $51-55$ & $45(84.91)$ & $8(15.09)$ & 1.24 & $0.5-3.04$ & \\
\hline \multicolumn{6}{|l|}{ Body mass index $\left(\mathrm{kg} / \mathrm{m}^{2}\right)$} \\
\hline$<18.5$ & $8(100)$ & $0(0)$ & 1 & & 0.414 \\
\hline $18.5-24.9$ & $156(85.71)$ & $26(14.29)$ & 1.55 & $0.19-12.71$ & \\
\hline $25-<30$ & $90(90.91)$ & $9(9.09)$ & 0.99 & $0.11-8.63$ & \\
\hline 30 & $48(84.21)$ & $9(15.79)$ & 1.84 & $0.21-16.17$ & \\
\hline \multicolumn{6}{|l|}{ Age of menarche (years) } \\
\hline$<12$ & $20(83.33)$ & $4(16.67)$ & 1.15 & $0.25-5.29$ & 0.654 \\
\hline $12-15$ & $259(87.8)$ & $36(12.2)$ & 0.8 & $0.26-2.45$ & \\
\hline$>15$ & $23(85.19)$ & $4(14.81)$ & 1 & & \\
\hline \multicolumn{6}{|l|}{ Waist circumference (cm) } \\
\hline$<80$ & $155(89.08)$ & $19(10.92)$ & 1 & & 0.313 \\
\hline 80 & 147 (85.47) & 25 (14.53) & 1.39 & $0.73-2.63$ & \\
\hline \multicolumn{6}{|l|}{ Parity } \\
\hline Nulliparous & $97(86.61)$ & $15(13.39)$ & 1.09 & $0.56-2.13$ & 0.794 \\
\hline Multiparous & $205(87.61)$ & 29 (12.39) & 1 & & \\
\hline \multicolumn{6}{|l|}{ History of contraceptive use } \\
\hline None & $179(86.89)$ & 27 (13.11) & 1 & & 0.921 \\
\hline Non-hormonal contraception & $109(87.2)$ & $16(12.8)$ & 0.97 & $0.5-1.89$ & \\
\hline Hormonal contraception & $14(93.33)$ & $1(6.67)$ & 0.47 & $0.06-3.77$ & \\
\hline \multicolumn{6}{|l|}{ Underlying disease } \\
\hline \multicolumn{6}{|l|}{ Hypertension } \\
\hline Yes & $35(81.4)$ & $8(18.6)$ & 1.70 & $0.73-3.95$ & 0.216 \\
\hline No & 267 (88.12) & $36(11.88)$ & 1 & & \\
\hline \multicolumn{6}{|l|}{ Diabetic Mellitus } \\
\hline Yes & $11(64.71)$ & $6(35.29)$ & 4.17 & $1.44-12.11$ & $0.004^{*}$ \\
\hline
\end{tabular}




\begin{tabular}{|c|c|c|c|c|c|}
\hline No & $291(88.45)$ & $38(11.55)$ & 1 & & \\
\hline \multicolumn{6}{|c|}{ Breast cancer } \\
\hline Yes & $9(81.82)$ & $2(18.18)$ & 1.55 & $0.32-7.44$ & 0.637 \\
\hline No & $293(87.46)$ & $42(12.54)$ & 1 & & \\
\hline \multicolumn{6}{|c|}{ Bleeding pattern } \\
\hline HMB & 155 (99.09) & $8(4.91)$ & 1 & & $<0.001^{*}$ \\
\hline IMB & $132(80)$ & $33(20)$ & 4.84 & $2.11-11.1$ & \\
\hline HPMB & $15(83.33)$ & $3(16.67)$ & 3.87 & $0.91-16.5$ & \\
\hline \multicolumn{6}{|c|}{ Endometrial thickness } \\
\hline$<10 \mathrm{~mm}$ & $196(94.69)$ & $11(5.31)$ & 1 & & $<0.001^{*}$ \\
\hline $10 \mathrm{~mm}$ & $66(69.47)$ & $29(30.53)$ & 7.53 & $3.52-17.4$ & \\
\hline \multicolumn{6}{|c|}{ Serum hormone levels } \\
\hline \multicolumn{6}{|c|}{ FSH (IU/mL) } \\
\hline$<10$ & $171(88.14)$ & $23(11.86)$ & 1 & & 0.699 \\
\hline $10-25$ & $53(88.33)$ & 7 (11.67) & 0.98 & $0.39-2.42$ & \\
\hline$>25$ & $42(75)$ & $14(25)$ & 1.33 & $0.65-2.74$ & \\
\hline \multicolumn{6}{|c|}{ Estradiol $(\mathrm{pg} / \mathrm{mL})$} \\
\hline$<100$ & $177(86.34)$ & $28(13.66)$ & 1 & & 0.137 \\
\hline 100 & $96(92.31)$ & $8(7.69)$ & 0.52 & $0.23-1.21$ & \\
\hline
\end{tabular}

Table 3: Factors associated with endometrial hyperplasia and endometrial cancer in perimenopausal women presenting with abnormal uterine bleeding: univariate analysis.

Non-BEH includes other benign causes of uterine bleeding, $\mathrm{BEH}^{+}$includes benign hyperplasia, atypical endometrial hyperplasia, endometrial intraepithelial neoplasia, endometrial cancer, HMB: Heavy Menstrual Bleeding; IMB; Intermenstrual Bleeding; HPMB: Heavy and Prolonged Menstrual Bleeding; FSH: Follicular Stimulating Hormone.

\begin{tabular}{|l|c|c|c|}
\hline \multicolumn{1}{|c|}{ Factors } & OR & 95\% CI & P-value \\
\hline Intermenstrual bleeding & 4.66 & $2.05-10.57$ & $<0.001^{*}$ \\
\hline Endometrial thickness $10 \mathrm{~mm}$ & 7.61 & $3.52-16.45$ & $<0.001^{*}$ \\
\hline
\end{tabular}

Table 4: Factors associated with endometrial hyperplasia and endometrial cancer in perimenopausal women presenting with abnormal uterine bleeding: multivariate logistic regression analysis.

* Statistically significant as p-value $<0.05$.

The uterine fibroid was the most common cause of the perimenopausal bleeding, accounting for 108 of 346 cases (31.21\%), which included submucous fibroids and intramural fibroids in size of diameter more than $4 \mathrm{~cm}$. Those lesions affect uterine bleeding, especially HMB pattern (69 of 108 cases), resulting in decreased hemoglobin levels and anemia affecting the quality of life, as previously reported by Foth D., et al. [12] and Puri K., et al. [13]. The adenomyosis, a disorder which endometrial gland and stroma involved in the myometrium, was the second common cause of perimenopausal bleeding, those found 84 of 346 cases (24.78\%), and also resulting in HMB pattern for 54 of 84 cases (64.29\%), which was similar to Struble J., et al. study [14] which discovered HMB presented in $50 \%$ of adenomyosis patient. And the result was relevant with Singh K., et al. study [15], which reported the common etiologies of 300 reproductive women presenting with AUB, in which $56 \%$ of participants were perimenopausal women, were uterine fibroid (30\%) and adenomyosis (29.66\%). However, the previous study by Van Den Bosch., et al. [5], performed a prospective study of 1217 women, which categorized 455 postmenopausal women, 672 premenopausal, and 93 perimenopausal women. They reveal the common causes of perimenopausal bleeding were hormonal imbalance, atrophic endometrium or endometrial polyp. As well as, Sun Y., et al. report most common non-structural and structural causes of AUB among reproductive women were ovulatory dysfunction (57.7\%) and endometrial polyps (16.2\%), respectively, followed by uterine fibroids (12\%) and uterine adenomyosis (4.94\%) [16]. The different results might be, that our participants are menopausal women only, not the reproductive women, and almost half of the participants (164/346) were indicated to hysterectomy, similarly the Singh K., et al. study [15]. The prevalence of endometrial hyperplasia and endometrial intraepithelial neoplasia (EIN) were 
found among 32 patients (9.25\%) which correlated with previous studies of 6-35\%. In addition, the prevalence of endometrium cancer of $3.47 \%$, which in line with prior reports of $1.1-9.67 \%$ in perimenopausal women presenting with AUB [5,15,17-19]. The bleeding patterns of IMB (previously defined as metrorrhagia) and HMB (previously defined as menorrhagia) were the common AUB patterns among perimenopausal women [17], as same as, Kazemijaliseh H., et al. [20] reported common bleeding characteristics of AUB in reproductive age women were metrorrhagia and intermenstrual bleeding, or FIGO definition of IMB $(23.2 \%)$ and heavy periods, or FIGO definition of HMB (15.2\%) with a more encountered among early and late reproductive years. Additionally, the studies of perimenopausal women presenting with AUB by Damle RP., et al. and Rezk M., et al. reported the most common bleeding pattern was menorrhagia or HMB of $48.86 \%$ and $67.5 \%$ [17,21], respectively followed by metrorrhagia, or IMB of $31.55 \%$ and $22.5 \%$, respectively.

The risk factors of endometrial cancer, majority (80 - 90\%) type I or endometrioid differentiation, are related to unopposed estrogen exposure effected on the hyperplasia- carcinoma endometrium sequence including obesity, history of the polycystic ovarian syndrome, anovulation history, early menarche, late menopause, tamoxifen therapy, nulliparity, and infertility. Other established risk factors, were not related to the unopposed estrogen, include older age more than 50 years, hypertension, obesity, type 2-DM, gall bladder disease, thyroid disease, Lynch syndrome, and BRCA mutation carriers $[10,22,23]$. However, there is still no established report of relevant risk factors of endometrial hyperplasia and cancer among perimenopausal women presenting with AUB. From the univariate analysis, the significant associating factors of endometrial hyperplasia and cancer are DM, intermenstrual bleeding pattern and endometrial thickness more than $10 \mathrm{~mm}$ from ultrasonography. However, after multiple logistic regression analyses, this study demonstrated two important risk factors among perimenopausal women presenting with AUB. First, the bleeding pattern IMB has a high association with the premalignant and malignant endometrium lesion (OR $=4.66,95 \% \mathrm{CI}: 2.05$ - 10.57). The result is related to a systematic review by Pennant ME., et al. which reported the prevalence rate of endometrial hyperplasia and cancer in perimenopausal patients presented with IMB pattern 5-times greater than those with HMB pattern [24]. The second significant risk associated factor was endometrial thickness $\geq 10 \mathrm{~mm}$ from sonographic findings. Moreover, this factor was also the highest risk for endometrial hyperplasia and cancer (OR = 7.61, 95\%CI: 3.52 - 16.45). According to the American College of Obstetricians and Gynecologists has purposed the thickness of endometrium $\leq 4 \mathrm{~mm}$ would be safe for postmenopausal women with uterine bleeding from endometrial hyperplasia and cancer [25]. Nevertheless, no clear cut of endometrial thickness in perimenopausal women, who still have cyclic menstrual shedding, was defined. Tsuda H., et al. reported a normal endometrial thickness in reproductive women could range from 2.8 to $16.5 \mathrm{~mm}$ in normal menstruation cycle and up to $24.4 \mathrm{~mm}$ in women with polymenorrhea [26]. The practice guideline from the Royal Thai College of Obstetrics and Gynecology recommends endometrial biopsy to exclude endometrial hyperplasia and cancer in the reproductive age women presenting with AUB when the endometrial thickness of $10-12 \mathrm{~mm}$ by transvaginal sonography. And, also supported by studies of perimenopausal women presenting with AUB, including Getpook C., et al. and Nicula R., et al. those reveal a high risk of endometrial hyperplasia and cancer in those with endometrial thickness more than $8 \mathrm{~mm}$ from the sonographic finding $[27,28]$ Despite the inconclusive cut-point of the endometrial thickness in perimenopausal women for predicting endometrial cancer, transvaginal sonography is still a valuable and available screening device for early detection of the precancerous and early-stage endometrial cancer.

\section{Conclusion}

The common etiologies of perimenopausal bleeding were uterine fibroids and adenomyosis, which commonly presented with intermenstrual or heavy menstrual bleeding. And the associated factors of endometrial hyperplasia and cancer in perimenopausal women presenting with AUB were IMB and thick endometrium by transvaginal sonography.

\section{Conflict of Interest}

The authors have no conflicts of interest in connection with this article.

\section{Bibliography}

1. Singh Sukhbir., et al. "Abnormal uterine bleeding in pre-menopausal women". Journal of Obstetrics and Gynaecology Canada: JOGC = Journal d'obstetrique et gynecologie du Canada: JOGC 35.5 (2013): 473-475.

2. Fraser Ian S., et al. "The FIGO recommendations on terminologies and definitions for normal and abnormal uterine bleeding". Seminars in Reproductive Medicine 29.5 (2011): 383-390.

3. Munro Malcolm G., et al. "FIGO classification system (PALMCOEIN) for causes of abnormal uterine bleeding in nongravid women of reproductive age". International Federation of Gynaecology and Obstetrics 113.1 (2011): 3-13.

4. Harlow Siobán D., et al. "Executive summary of the Stages of Reproductive Aging Workshop + 10: addressing the unfinished agenda of staging reproductive aging". The Journal of Clinical Endocrinology and Metabolism 97.4 (2012): 1159-1168. 
5. Van den Bosch, T., et al. "Intra-cavitary uterine pathology in women with abnormal uterine bleeding: a prospective study of 1220 women". Facts, Views and Vision in ObGyn 7.1 (2015): 17-24.

6. American College of Obstetricians and Gynecologists. "ACOG committee opinion no. 557: Management of acute abnormal uterine bleeding in nonpregnant reproductive-aged women". Obstetrics and Gynecology 121.4 (2013): 891-896.

7. Ferlay Jacques., et al. "Cancer incidence and mortality worldwide: sources, methods and major patterns in GLOBOCAN 2012". International Journal of Cancer 136.5 (2015): E359386.

8. Reed Susan D., et al. "Incidence of endometrial hyperplasia". American Journal of Obstetrics and Gynecology 200.6 (2009): 678.e1-e6.

9. Constantine Ginger D., et al. "Increased Incidence of Endometrial Cancer Following the Women's Health Initiative: An Assessment of Risk Factors". Journal of Women's Health (2002) 28.2 (2019): 237-243.

10. Ali Aus Tariq. "Risk factors for endometrial cancer". Ceska Gynekologie 78.5 (2013): 448-459.

11. Munro Malcolm G., et al. "The two FIGO systems for normal and abnormal uterine bleeding symptoms and classification of causes of abnormal uterine bleeding in the reproductive years: 2018 revisions". International Federation of Gynaecology and Obstetrics 143.3 (2018): 393-408.

12. Foth Dolores., et al. "Symptoms of uterine myomas: data of an epidemiological study in Germany". Archives of Gynecology and Obstetrics 295.2 (2017): 415-426.

13. Puri Kanchan., et al. "Submucosal fibroids and the relation to heavy menstrual bleeding and anemia". American Journal of Obstetrics and Gynecology 210.1 (2014): 38.e1-7.

14. Struble Jennifer., et al. "Adenomyosis: A Clinical Review of a Challenging Gynecologic Condition". Journal of Minimally Invasive Gynecology 23.2 (2016): 164-185.

15. Singh Kanika., et al. "A Clinicopathological Correlation of International Federation of Gynecology and Obstetrics's PALMCOEIN Classification of Abnormal Uterine Bleeding: Indian Scenario". Journal of Mid-Life Health 10.3 (2019): 147-152.

16. Sun Yu., et al. "Prevalence of abnormal uterine bleeding according to new International Federation of Gynecology and Obstetrics classification in Chinese women of reproductive age: A cross-sectional study". Medicine 97.31 (2018): e11457.

17. Damle Rajshri P., et al. "Clinicopathological Spectrum of Endometrial Changes in Peri-menopausal and Post-menopausal Abnormal Uterine Bleeding: A 2 Years Study". Journal of Clinical and Diagnostic Research: JCDR 7.12 (2013): 2774-2776.
18. Abid Mariam., et al. "Clinical pattern and spectrum of endometrial pathologies in patients with abnormal uterine bleeding in Pakistan: need to adopt a more conservative approach to treatment". BMC Women's Health 14.132 (2014).

19. Doraiswami Saraswathi., et al. "Study of endometrial pathology in abnormal uterine bleeding". Journal of Obstetrics and Gynaecology of India 61.4 (2011): 426-430.

20. Kazemijaliseh Hadigheh., et al. "A Population-Based Study of the Prevalence of Abnormal Uterine Bleeding and its Related Factors among Iranian Reproductive-Age Women: An Updated Data". Archives of Iranian Medicine 20.9 (2017): 558-563.

21. Rezk M., et al. "Perimenopausal bleeding: Patterns, pathology, response to progestins and clinical outcome". Institute of $\mathrm{Ob}$ stetrics and Gynaecology 35.5 (2015): 517-521.

22. "Practice Bulletin No. 149: Endometrial cancer". Obstetrics and Gynecology 125.4 (2015): 1006-1026.

23. Braun Michael M., et al. "Diagnosis and Management of Endometrial Cancer". American Family Physician 93.6 (2016): 468474.

24. Pennant ME., et al. "Premenopausal abnormal uterine bleeding and risk of endometrial cancer". BJOG: An International Journal of Obstetrics and Gynaecology 124.3 (2017): 404-411.

25. "ACOG Committee Opinion No. 734: The Role of Transvaginal Ultrasonography in Evaluating the Endometrium of Women With Postmenopausal Bleeding". Obstetrics and Gynecology 131.5 (2018): e124-e129.

26. Tsuda, Hiroshi., et al. "Measurement of endometrial thickness in premenopausal women in office gynecology". Reproductive Medicine and Biology 17.1 (2017): 29-35.

27. Nicula Renata., et al. "Accuracy of transvaginal ultrasonography compared to endometrial biopsy for the etiological diagnosis of abnormal perimenopausal bleeding". Clujul Medical (1957) 90.1 (2017): 33-39.

28. Getpook Chatpavit and Saranya Wattanakumtornkul. "Endometrial thickness screening in premenopausal women with abnormal uterine bleeding". The Journal of Obstetrics and Gynaecology Research 32.6 (2006): 588-592.

\section{Assets from publication with us}

- Prompt Acknowledgement after receiving the article

- Thorough Double blinded peer review

- Rapid Publication

- Issue of Publication Certificate

- High visibility of your Published work

Website: www.actascientific.com/

Submit Article: www.actascientific.com/submission.php

Email us: editor@actascientific.com

Contact us: +919182824667 